\title{
Advances in solid-catalytic and non-catalytic technologies for biodiesel production
}

\begin{abstract}
The insecure supply of fossil fuel coerces the scientific society to keep a vision to boost investments in the renewable energy sector. Among the many renewable fuels currently available around the world, biodiesel offers an immediate impact in our energy. In fact, a huge interest in related research indicates a promising future for the biodiesel technology. Heterogeneous catalyzed production of biodiesel has emerged as a preferred route as it is environmentally benign needs no water washing and product separation is much easier. The number of well-defined catalyst complexes that are able to catalyze transesterification reactions efficiently has been significantly expanded in recent years. The activity of catalysts, specifically in application to solid acid/base catalyst in transesterification reaction depends on their structure, strength of basicity/acidity, surface area as well as the stability of catalyst. There are various process intensification technologies based on the use of alternate energy sources such as ultrasound and microwave. The latest advances in research and development related to biodiesel production is represented by non-catalytic supercritical method and focussed exclusively on these processes as forthcoming transesterification processes. The latest developments in this field featuring highly active catalyst complexes are outlined in this review. The knowledge of more extensive research on advances in biofuels will allow a deeper insight into the mechanism of these technologies toward meeting the critical energy challenges in future.
\end{abstract}

Keyword: Biodiesel; Catalyst; Heterogeneous; Supercritical 